\title{
O Acesso Avançado como estratégia de organização da agenda e de ampliação do acesso em uma Unidade Básica de Saúde de Estratégia Saúde da Família, município de Diadema, São Paulo
}

\author{
The Advanced Access as a strategy to organize the agenda and increase access in a Basic \\ Health Unit of Family Health Strategy, Diadema, São Paulo, Brazil
}

\section{Acceso Avanzado como estrategia para organizar la agenda y ampliar el acceso en una Unidad Básica de Salud de la Estrategia Salud de la Familia, Diadema, São Paulo, Brasil}

Ferla Maria Simas Bastos Cirino', Douglas Augusto Schneider Filho', Lucia Yasuko Izumi Nichiata², Lislaine Aparecida Fracolli²

1 Secretaria Municipal de Saúde de Diadema, Diadema, São Paulo, Brasil

2 Universidade de São Paulo, São Paulo, São Paulo, Brasil

\section{Resumo}

Introdução: Pressionadas pela demanda e por agendas lotadas, equipes de saúde têm discutido estratégias de reorganização da agenda e ampliação do acesso. O Acesso Avançado vem ganhando espaço nesta discussão como uma estratégia desenvolvida nos Estados Unidos, que tem como princípio "Faça hoje o trabalho de hoje!". Propôs-se descrever a influência da implantação do Acesso Avançado sobre acesso ao atendimento de usuários numa Unidade de Saúde da Família no município de Diadema. Métodos: Tratou-se de um relato de experiência da gerente da unidade no processo de implantação do Acesso Avançado, com a proposta de analisar as agendas e os relatórios de produção dos profissionais de saúde (médicos, enfermeiros e técnicos de enfermagem) no período de janeiro de 2017 a abril de 2018 e apresentar estatística descritiva da influência do Acesso Avançado sobre os atendimentos desses profissionais. O Acesso Avançado foi amplamente discutido com a equipe e com o conselho gestor, num processo de construção conjunta de fluxos e novas agendas. Resultados: Identificou-se aumento de $157 \%$ no número de usuários atendidos em consultas, passando de 1.048 em janeiro de 2017 para 2.694 atendimentos em abril de 2018, e queda de $13 \%$ para $2 \%$ na taxa de faltas dos usuários às consultas. Conclusão: Pôde-se demonstrar que a estratégia foi efetiva na ampliação do acesso ao serviço de saúde, gerando também efeitos positivos na organização da agenda e dos processos de trabalho das equipes. Espera-se contribuir e fomentar discussões acerca da otimização do acesso à Atenção Primária à Saúde (APS), suas complexidades e peculiaridades, e propor análise da influência do Acesso Avançado sobre os demais atributos da APS, particularmente nos atributos de longitudinalidade e integralidade do cuidado.

Descritores: Acesso aos Serviços de Saúde; Qualidade, Acesso e Avaliação da Assistência à Saúde; Atenção Primária à Saúde; Assistência Integral à Saúde.

Como citar: Cirino FMSB, Schneider Filho DA, Nichiata LYI, Fracolli LA. O Acesso Avançado como estratégia de organização da agenda e de ampliação do acesso em uma Unidade Básica de Saúde de Estratégia Saúde da Família, município de Diadema, São Paulo. Rev Bras Med Fam Comunidade. 2020;15(42):2111. https://doi.org/10.5712/rbmfc15(2)2111

\author{
Autor correspondente: \\ Ferla Maria Simas Bastos Cirino. \\ E-mail: ferlacirino@hotmail.com \\ Fonte de financiamento: \\ declaram não haver. \\ Parecer CEP: \\ não se aplica. \\ Procedência e revisão por pares: \\ revisado por pares. \\ Recebido em: 18/06/2019 \\ Aprovado em: 01/11/2019
}




\begin{abstract}
Introduction: Pressured by demand and busy schedules, health teams have been discussing strategies for reorganizing the agenda and expanding access. Advanced Access has been gaining ground in this discussion as a strategy developed in the United States that has the principle "Do today's work today!". It was proposed to describe the influence of the implementation of Advanced Access on access to user care Advanced Access in a Family Health Unit in the city of Diadema. Methods: This was an experience report of the unit manager in the process of implementation of Advanced Access, with the proposal to analyze the agendas and production reports of health professionals (doctors, nurses and nursing technicians) during the period. January 2017 to April 2018 and present descriptive statistics of the influence of Advanced Access on the care of these professionals. Advanced Access has been extensively discussed with staff and management council in a process of jointly building flows and new agendas. Results: There was a 157\% increase in the number of users served in consultations, from 1,048 in January 2017 to 2,694 calls in April 2018 , and a drop from $13 \%$ to $2 \%$ in the rate of missed users. Conclusion: It was demonstrated that the strategy was effective in expanding access to health services, also generating positive effects on the organization of the agenda and work processes of teams. It is expected to contribute and foster discussions about the optimization of access to Primary Health Care (PHC), its complexities and peculiarities, and propose analysis of the influence of Advanced Access on the other attributes of PHC, particularly on the longitudinality and comprehensiveness attributes of care.
\end{abstract}

Keywords: Health Services Accessibility; Health Care Quality; Access, and Evaluation; Primary Health Care; Comprehensive Health Care.

\title{
Resumen
}

Introducción: Presionado por la demanda y los horarios ocupados, los equipos de salud han estado discutiendo estrategias para reorganizar la agenda y ampliar el acceso. Advanced Access ha ido ganando terreno en esta discusión como una estrategia desarrollada en los Estados Unidos que tiene el principio "¡Haz el trabajo de hoy hoy!". Se propuso describir la influencia de la implementación del Acceso avanzado en el acceso a la Atención al usuario Acceso avanzado en una Unidad de salud familiar en la ciudad de Diadema. Método: Este fue un informe de experiencia del gerente de la unidad en el proceso de implementación de Acceso Avanzado, con la propuesta de analizar las agendas y los informes de producción de los profesionales de la salud (médicos, enfermeras y técnicos de enfermería) durante el período. Enero de 2017 a abril de 2018 y presentar estadísticas descriptivas de la influencia de Advanced Access en la atención de estos profesionales. El acceso avanzado ha sido ampliamente discutido con el personal y el consejo de administración en un proceso de construcción conjunta de flujos y nuevas agendas. Resultados: Hubo un aumento del $157 \%$ en el número de usuarios atendidos en las consultas, de 1.048 en enero de 2017 a 2.694 llamadas en abril de 2018 , y una caída del $13 \%$ al $2 \%$ en la tasa de usuarios perdidos. Conclusión: Se demostró que la estrategia fue efectiva para expandir el acceso a los servicios de salud, generando también efectos positivos en la organización de la agenda y los procesos de trabajo de los equipos. Se espera que contribuya y fomente discusiones sobre la optimización del acceso a la Atención Primaria de Salud (APS), sus complejidades y peculiaridades, y proponga el análisis de la influencia del Acceso Avanzado en los otros atributos de la APS, particularmente en los atributos de longitudinalidad e integralidad de la atención.

Palabras clave: Accesibilidad a los Servicios de Salud; Calidad, Acceso y Evaluación de la Atención de Salud; Atención Primaria de Salud; Atención Integral de Salud.

\section{INTRODUÇÃO}

Garantir o acesso aos cuidados primários à saúde, em tempo oportuno, é um dos maiores desafios dos sistemas públicos de saúde, tanto no Brasil quanto em outros países. ${ }^{1}$ Uma Atenção Primária à Saúde (APS) forte e resolutiva depende de acesso facilitado, a fim de garantir o atendimento em tempo oportuno, no horário mais adequado, com agendamento confortável e onde o usuário seja atendido por sua equipe de referência. ${ }^{2}$

A qualidade do acesso à APS é avaliada internacionalmente por duas medidas operacionais: o acesso oportuno e a continuidade do cuidado. Considera-se acesso oportuno a capacidade de se obter um agendamento tão rápido quanto possível, oportunizando atendimento às demandas e condições agudas, que poderiam gerar gastos desnecessários se desviadas aos serviços de urgência e emergência. A continuidade do cuidado é alcançada quando o profissional de saúde de referência mantém o acompanhamento do usuário sempre que necessário, visando o fortalecimento de uma relação duradoura entre este e o profissional e, consequentemente, intensificando o cuidado individual e holístico. ${ }^{3}$

O presente artigo foca no primeiro aspecto, que trata do acesso oportuno ao atendimento em serviços de saúde, por meio das consultas com profissionais de saúde. 
A literatura internacional aponta que a crise econômica instalada nas últimas décadas demarca a existência de barreiras dos usuários aos serviços de saúde, que se traduz em filas para marcação de consultas e atendimentos. ${ }^{4}$

Desde a década de 1990 a APS vem se fortalecendo como porta de acesso preferencial ao Sistema Único de Saúde (SUS), "devendo receber e ouvir todas as pessoas que procuram seus serviços, de modo universal, de fácil acesso e sem diferenciações excludentes, e a partir daí construir respostas para suas demandas e necessidades". ${ }^{5}$

No Brasil, a Constituição de 1988 assegura a saúde como um direito universal e um dever do Estado, porém, mesmo com todos os avanços conquistados, ainda convivemos com a realidade desigual e excludente do acesso ao SUS. Há um entendimento de que o acesso à APS não ocorre de forma tão facilitada quanto deveria. São muitos os fatores que dificultam esse acesso: cobertura insuficiente da ESF; desequilíbrio entre demanda e oferta; distribuição inadequada do território; restrição de acesso por horário reduzido de funcionamento dos serviços que incompatibiliza o acesso de usuários que trabalham em horário convencional; tempo de espera prolongado para marcação de consultas e o número excessivo de usuários nas equipes. ${ }^{6-8}$ Desta forma, a acessibilidade é a dimensão de menor satisfação do usuário da $E S F, 9,10$ e o atributo com maior avaliação negativa na visão dos profissionais de saúde. ${ }^{11}$

Diante deste cenário e pressionados pela demanda e por agendas lotadas, os serviços de saúde têm adotado o Acesso Avançado como estratégia de organização da agenda e de ampliação do acesso, tanto no Brasil como fora do país. ${ }^{2}$ A primeira experiência ocorreu em 1990 na Califórnia ${ }^{12,13}$, Estados Unidos da América (EUA), e nos anos 2000 no Reino Unido ${ }^{14}$, bem como em outros países da Europa ${ }^{2}$. No Brasil, as primeiras iniciativas partiram de algumas unidades de Florianópolis (SC) em $2007^{15}$ e de São Paulo (SP) e Rio de Janeiro (RJ) entre os anos de 2013 e $2014 .{ }^{2}$ Neste mesmo período, a Secretaria Municipal de Saúde de Curitiba (PR) adotou o Acesso Avançado como forma de organizar e qualificar o acesso em toda sua rede básica de saúde. ${ }^{2}$

O Acesso Avançado tem uma única regra: "Faça o trabalho de hoje, hoje!". É uma forma de organização do serviço que permite que pessoas busquem a unidade por quaisquer problemas de saúde, seja ele urgente, de rotina ou prevenção. Este sistema preconiza que os usuários sejam atendidos no mesmo dia ou em até 48 horas, favorecendo a continuidade do cuidado, reduzindo as faltas e diminuindo significativamente as filas, sem recursos adicionais. ${ }^{12,13}$

O Acesso Avançado tenta fechar a lacuna existente entre a oferta e a demanda. Para isso, adota uma estratégia oposta e inerente a outros modelos de agendamento. Enquanto os outros modelos empurram uma grande parte do trabalho de hoje para o futuro, o modelo de Acesso Avançado protege a capacidade futura trazendo todo o trabalho atual para o tempo presente..$^{13}$

$\mathrm{Na}$ Inglaterra ${ }^{14}$ e nos EUA ${ }^{12}$, estudos demonstram que este modelo vem se mostrando eficiente, ocasionando maior satisfação das equipes por sentirem-se mais resolutivas, e dos usuários por terem suas necessidades atendidas em tempo oportuno. . $^{2,12,13,14}$ São escassos os estudos nacionais que avaliam modelos de agendamento e melhoria do acesso à APS. ${ }^{1}$

$O$ presente relato tem como objetivo descrever a influência sobre o acesso às consultas na unidade de saúde a partir da implementação do Acesso Avançado como estratégia de organização da agenda em uma Unidade Básica de Saúde (UBS) que opera a ESF no município de Diadema, São Paulo. 


\section{MÉTODOS}

Tratou-se de um relato de experiência vivenciada por uma das autoras, responsável por propor e por implementar o Acesso Avançado como estratégia de ampliação do acesso e organização das agendas, àquela época, gerente da UBS (microcenário desse relato).

\section{Macrocenário da Experiência}

Diadema possui uma população, estimada pelo IBGE em 2018, de 420.934 habitantes, distribuídos em uma área de 30,7 km², 100\% urbanizada e está inserida na Sub-região do Grande ABC. Possui a segunda maior densidade demográfica do país, com $13,7 \mathrm{hab} / \mathrm{m}^{2}$. Apresenta uma população relativamente jovem, com menor índice de envelhecimento da sua sub-região $(51,16 \%)$. Mais de $20 \%$ da população apresenta vulnerabilidade social alta ou muito alta, onde $10,5 \%$ da população vive em aglomerados urbanos subnormais. ${ }^{16}$

Em outubro de 2019, o município possuía 20 UBS dispostas em seu território, todas atuando com modelo da ESF. A cidade conta com 96 equipes de Saúde da Família, sendo cada equipe responsável por cerca de 4.300 pessoas. A ESF cobre $100 \%$ da população do município e estima-se que cerca de $80 \%$ da população utiliza intensivamente o SUS, com pequenas variações regionais.

\section{Microcenário da Experiência}

A UBS Jardim ABC, local do estudo, opera com o modelo da ESF desde 2008, trabalhando com quatro equipes de Saúde da Família, compostas por um médico generalista, um enfermeiro, dois técnicos de enfermagem e cinco agentes comunitários de saúde. A unidade tem sob sua responsabilidade cerca de 18.000 pessoas que residem em sua área de abrangência, presta atendimento de segunda a sexta-feira, das 07 h00 às 17 h00 e está localizada na zona norte da cidade, bairro do Taboão.

\section{Descrição da Experiência: processo de implantação do Acesso Avançado}

A UBS Jardim ABC foi pioneira da implantação no município, por meio de iniciativa da gerente da UBS que adotou o Acesso Avançado com objetivo de organizar a agenda e ampliar o acesso da população aos serviços oferecidos pela UBS. Antes de abril de 2017, quando iniciada a implantação do Acesso Avançado, a unidade trabalhava com o modelo de agendamento tradicional, fragmentada em função de grupos programáticos. Agendas médicas com vagas que se esgotavam entre 2 e 5 dias após abertura na recepção, com datas para consultas entre 30 e 40 dias. As agendas das enfermeiras estavam sempre vazias, igualmente fragmentadas por grupos programáticos e grande parte das vagas preenchida pela coleta de exame de Papanicolau e puericultura.

$\mathrm{O}$ atendimento da demanda espontânea com queixa aguda era direcionado para o acolhimento e a escuta era realizada por uma enfermeira, durante todo o período de funcionamento da UBS. Eram poucas vagas de encaixe do dia, utilizadas de acordo com a triagem. Os usuários do serviço eram direcionados para o médico que tinha vaga disponível naquele momento e não para o seu médico de equipe de sua referência. 
Havia constantes tensionamentos quando as vagas se esgotavam, tanto entre a enfermeira do acolhimento e o usuário cuja vaga fora negada, quanto entre aquela profissional e o médico, sobretudo em razão de não ter vagas suficientes para os atendimentos necessários. Não havia um fluxo bem definido e não existia um protocolo de demanda espontânea. O usuário do serviço que não conseguia a vaga do dia era direcionado a voltar na data de abertura da agenda médica, que acontecia sempre no início de cada mês.

Era forte o descontentamento da gerente da UBS e das equipes quanto ao modelo hegemônico em que operava uma agenda na recepção, bem como quanto às barreiras de acesso que permeavam este modelo tradicional de agendamento. Diante disso, foram realizadas diversas rodas de conversa com a equipe para sensibilização e discussão sobre as diferentes formas de acesso descritas na literatura nacional e internacional, até a decisão de implantação do Acesso Avançado, criando espaços de construção conjunta e horizontal.

A gerente contou com o apoio do colegiado de gestão da UBS para o desenho dos novos fluxos e agendas. O colegidado de gestão da UBS era composto por representantes de cada categoria e setores da unidade (um médico, uma enfermeira, uma dentista, uma auxiliar de saúde bucal, uma técnica de enfermagem, um agentes comunitárias de saúde de cada equipe, uma assistente social, um representante da recepção, um representante da central de regulação e uma representante do serviço de limpeza), com um cronograma de reuniões semanais, para discutir a ampliação do acesso aos serviços na UBS. O Acesso Avançado foi amplamente discutido em todos os espaços coletivos existentes na unidade e com o Conselho Gestor, no período de fevereiro a abril de 2017. O Conselho Gestor era composto por quatro representantes da população, dois representantes do seguimento trabalhador da UBS e a gerente como representante da gestão.

As discussões objetivavam não apenas a sensibilização da equipe, mas, principalmente, promover um espaço de reflexão crítica cuja nova proposta não trazia apenas um novo modelo de agenda, como também uma reorganização de processos de trabalhos.

Nessas discussões também permeavam questões relacionadas à importância em se assegurar os princípios da ESF, garantindo espaços de fortalecimento por meio da educação permanente e resgatando o protagonismo do enfermeiro na APS, reforçando na equipe o entendimento de que a APS é um espaço privilegiado de construção de vínculos, gestão do cuidado e atenção para o indivíduo, a sua família e a comunidade, que tem necessidades de saúde social e historicamente construídas e que precisam de um olhar diferenciado neste sentido de todos os membros da equipe multiprofissional.

O processo de implantação do Acesso Avançado na UBS Jardim ABC foi gradativo, iniciado com o projeto-piloto da equipe identificada como "Equipe B", em abril de 2017 e, nos meses subsequentes, nas demais equipes, "Equipe A", "Equipe C" e "Equipe D". A partir de julho de 2017, a UBS operou com o Acesso Avançado para os usuários de todas as suas quatro equipes.

Os fluxos foram redesenhados com bases nos protocolos de enfermagem do município de Diadema e nos Cadernos de Atenção Básica do Ministério da Saúde, em especial o de "Acolhimento à demanda espontânea". ${ }^{17}$ Todos os usuários passaram a ser encaminhados para suas respectivas equipes de referência para garantir o fortalecimento do vínculo e a continuidade do cuidado. 
O presente estudo toma como indicador do acesso às consultas na unidade de saúde: número percentual de atendimentos individuais e percentual de faltas dos usuários às consultas, em período anterior e posterior a implementação do Acesso Avançado.

Com a implantação do novo modelo, as agendas saíram da recepção e passaram a ser gerenciadas pela própria equipe da ESF. Todos os usuários que chegavam à UBS foram atendidos e avaliados no mesmo dia, por um ou mais membros da equipe, de acordo com suas demandas e necessidades, respeitando-se sempre as competências técnicas e os limites de atuação de cada profissional. A primeira escuta era, prioritariamente, realizada pelo técnico de enfermagem, porém todos os membros da equipe foram capacitados e sensibilizados para acolhimento e escuta qualificada.

As agendas tiveram padronização inicial, no primeiro mês. As agendas médicas desenhadas com disponibilidade de $50 \%$ das vagas para atender às consultas do dia (demanda espontânea), enquanto a outra metade foi destinada às consultas programadas/retornos. As agendas dos enfermeiros ficaram com $90 \%$ para demanda espontânea e 10\% para consultas programadas de puericultura e pré-natal. Do segundo mês em diante, todas as agendas médicas e de enfermeiros foram padronizadas com a proporção de $75 \%$ para atendimento à demanda espontânea e $25 \%$ para consultas programadas.

Nesta nova agenda foram garantidos os horários de reunião de equipe, único período em que o atendimento da equipe era suspenso, sendo as demandas agudas (urgência e emergência) direcionadas para outra enfermeira. A reunião de equipe era um espaço preservado para garantir que a equipe realizasse discussões e ajustes periódicos dos fluxos e dos processos de trabalho do novo modelo de agendamento. Durante as visitas domiciliares, faltas ou férias de um dos membros da equipe, as demandas espontâneas eram direcionadas a outro profissional da equipe presente na unidade, garantindo a continuidade do cuidado.

Como material empírico foram consultadas as agendas médicas e das enfermeiras e analisados os relatórios de produção de cada profissional (médicos, enfermeiras e técnicos de enfermagem), extraídos da plataforma e-SUS AB. Destes documentos foram extraídas as variáveis: números de agendamentos, números de faltas e o número de atendimentos individuais. Considerou-se atendimento individual todo o usuário que passou por atendimento individualizado por qualquer profissional da equipe (médico, enfermeiro ou técnico de enfermagem) e denominado consulta quando este atendimento foi realizado por médicos ou enfermeiros. Os relatórios do e-SUS AB considera como fonte de informação a "Ficha de Atendimento Individual" e os atendimentos realizados no Prontuário Eletrônico do Cidadão. Os dados coletados referem-se ao período de janeiro de 2017 a abril de 2018. Como o Acesso Avançado foi implantado no mês de abril de 2017 , os dados desse estudo dizem respeito a doze meses de utilização dessa estratégia na UBS.

O estudo obteve anuência da Secretaria Municipal da Saúde de Diadema e, embora se trate de fonte de dados secundários do e-SUS $A B$, encaminhou-se para análise do Comitê de Ética em Pesquisa da Escola de Enfermagem da Universidade de São Paulo, que não percebeu quaisquer empecilhos éticos ou legais para a coleta da base de dados deste estudo, dando parecer favorável sob $\mathrm{n}^{\circ}$ 3.285.854. Os pesquisadores assinaram termo de confidencialidade e sigilo dos dados. 


\section{RESULTADO}

Foi identificado aumento no número total de atendimentos individuais quando considerados todos os profissionais das equipes envolvidos (médicos, enfermeiros e técnicos de enfermagem) ao longo do período estudado. Em janeiro de 2017 foram realizados 1.048 atendimentos, passando para 2.694 atendimentos individuais em abril de 2018, como mostra o Gráfico 1. Desta forma, houve um aumento de $157 \%$ quando comparados os meses de janeiro de 2017 e abril de 2018. A média mensal de atendimento, calculada entre os meses de janeiro e março de 2017, que antecederam a implantação do Acesso Avançado, era de 1.262 atendimentos mensais, e nos meses após a implantação, entre abril de 2017 e abril de 2018, passou para uma média de 2.153 atendimentos mensais.

$\mathrm{Na}$ agenda médica identificou-se aumento de $36 \%$ no número de atendimentos quando comparados os meses de janeiro de 2017 e abril de 2018. O maior aumento foi observado nas consultas de enfermagem, que chegou a 792\%, passando de 125 atendimentos mensais em janeiro de 2017 para 1.115, em abril de 2018, com média de 211 atendimentos nos meses que antecederam (entre janeiro e março de 2017) e, nos meses após a implantação do Acesso Avançado (entre abril de 2017 e abril de 2018), uma média de 731 atendimentos. O número de atendimento em escuta inicial realizado pelos técnicos de enfermagem em acolhimento à demanda espontânea, que nos meses de janeiro a março de 2017 era nulo, passou a representar média mensal de 400 usuários atendidos entre abril de 2017 e abril de 2018.

Contabilizou-se, também, a redução no número de faltas dos usuários às consultas médicas, de $13 \%$ entre os meses de janeiro e março de 2017 para $2 \%$ em os meses de abril de 2017 e abril de 2018 (no período após a implantação), quase sempre observadas nas agendas programadas, visto que a demanda espontânea tinha seu atendimento assegurado para o dia ou em no máximo 48 horas, possibilitando-se quase zerar as faltas às consultas.

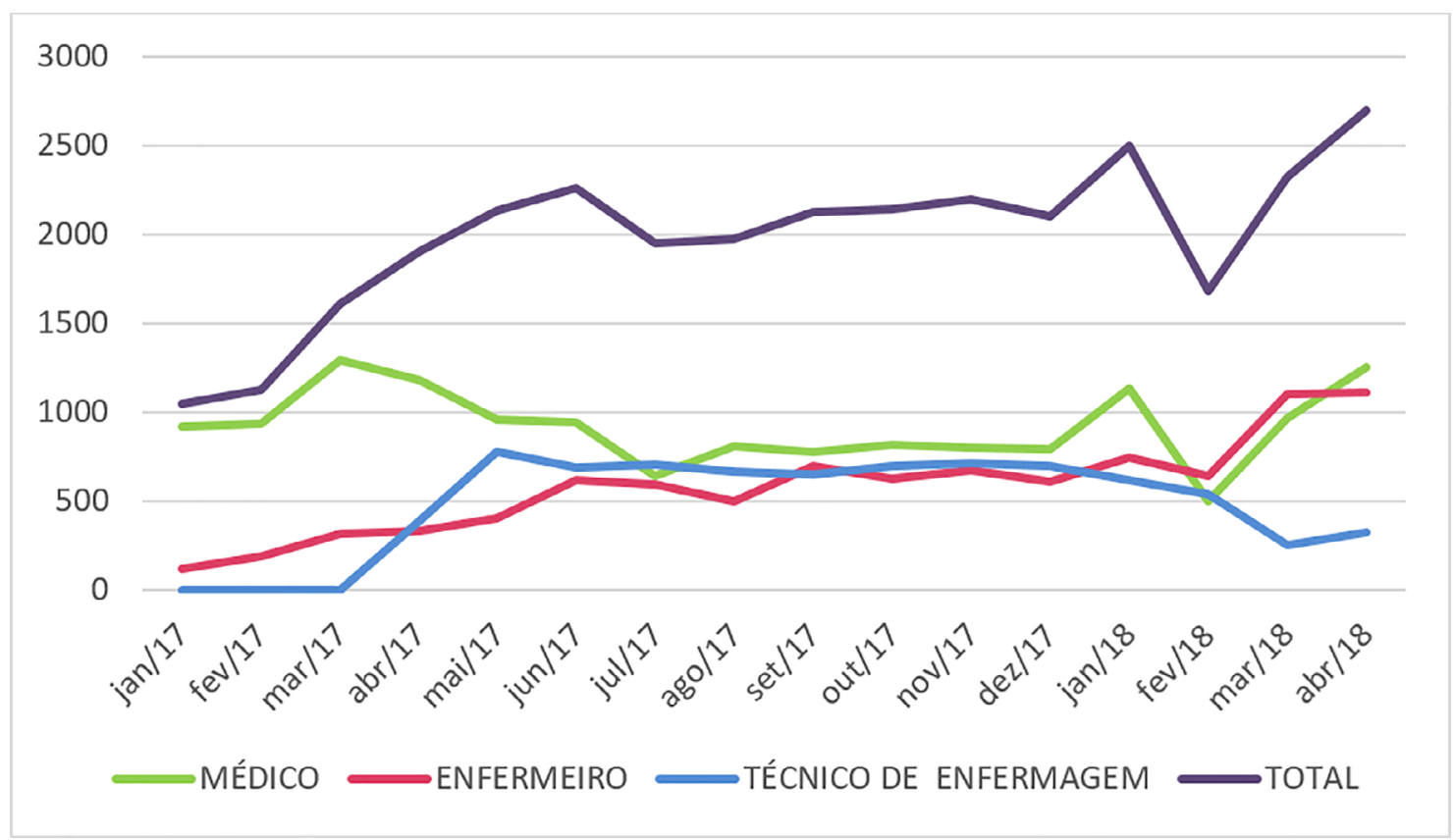

Gráfico 1. Número de atendimentos individuais segundo categoria profissional entre os meses de janeiro de 2017 e abril de 2018, UBS Jardim ABC, Diadema, São Paulo. 


\section{DISCUSSÃO}

O Acesso Avançado mostrou ser uma estratégia viável de ampliação do acesso e de organização da agenda. Após sua implantação, a UBS passou a atender o dobro de usuários em relação aos meses anteriores.

Ao promover o acesso à agenda da equipe multiprofissional, vislumbra-se romper com o modelo biomédico hegemônico, que privilegiava a agenda médica, aproximando-se dos pressupostos da ESF.

Observou-se que houve incremento nas agendas médicas a partir do mês anterior à implantação oficial do Acesso Avançado, em março de 2017. Esse achado pode ser explicado pelo fato das oficinas sobre a ampliação do acesso terem se iniciado um mês antes, com discussão sobre estratégias para reduzir os agendamentos futuros, e pequenas mudanças de processos de trabalho já podiam ser observadas, com redução das taxas de falta às consultas médicas, além das vagas produzidas pelas faltas serem oportunamente utilizadas para atender às demandas espontâneas como forma de reduzir a demanda reprimida, preparando o cenário para implantação do novo modelo.

As agendas das enfermeiras eram subutilizadas no modelo tradicional de agendamento, sempre vazias e fragmentadas. Com a mudança do modelo, os atendimentos tiveram um incremento superior ao dos médicos que já tinham agendas lotadas e com sua capacidade instalada utilizada, porém de forma fragmentada e, muitas vezes com demandas que poderiam ser direcionadas para outros membros da equipe multiprofissional.

A autonomia das enfermeiras, bem como a sua resolutividade diante das necessidades da população foram amplamente discutidas e reforçadas, entendendo que uma das ações mais importantes para melhorar o acesso na APS é o maior envolvimento do enfermeiro no cuidado das pessoas da sua área. ${ }^{2}$

As agendas médicas foram potencializadas para atender demandas reais e do momento, oportunizando a UBS como porta de entrada do sistema e a equipe de referência como responsável pela atenção ao primeiro contato.

Muitos usuários não conhecem detalhes técnicos para avaliar a gravidade do seu problema e o ponto de atenção que melhor responda às suas necessidades. A ausência de um ponto de entrada facilitado na APS faz com que a atenção adequada possa não ser obtida ou tenha sua atenção adiada, incindindo em gastos adicionais. ${ }^{18}$

Revisão integrativa da literatura sobre acesso aos cuidados primários de saúde aponta que todos os modelos de agendamento utilizados na APS apresentam vantagens e desvantagens. Um sistema de saúde menos iníquo provê o alcance de duas diretrizes da APS: acesso oportuno e continuidade do cuidado. Contudo, considera-se o Acesso Avançado um modelo propício para conseguir equilibrar a capacidade e a demanda, reduzindo simultaneamente o tempo de espera. ${ }^{1}$

No modelo de Acesso Avançado o atendimento à demanda espontânea assume o mesmo nível de relevância que as ações programática. A organização do trabalho passa a ser embasada no território e na população adscrita, incluindo as ações de prevenção/promoção e os cuidados clínicos, assumindo a dupla responsabilidade do cuidado individual e de saúde pública. ${ }^{15}$

Mesmo que a oferta de serviços de saúde seja insuficiente, pequenas mudanças no modelo de agendamento de consultas podem evitar filas e sofrimento humano. ${ }^{9}$ 
Todavia, estudos apontam a necessidade de se estabelecer estrutura para sustentar as mudanças de agendamento, bem como a inclusão da população na discussão, uma vez que há evidências de que a preferência do usuário orienta a demanda. ${ }^{1,19}$

O presente relato, embora tenha se dedicado a analisar o acesso ao atendimento no serviço de saúde, verificado por meio do acesso às consultas médicas e de enfermagem, outros estudos realizados em países como EUA e Inglaterra discutem que este modelo tem potência na direção da promoção da equidade, longitudinalidade e interdisciplinaridade. ${ }^{12,13,14}$

Esses estudos salientam que a promoção da equidade se dá na medida que é possível oportunizar acesso a novos usuários, com suas demandas atendidas e com resposta de um profissional adequado para respondê-las. A longitudinalidade do cuidado é garantida quando o Acesso Avançado permite que o usuário seja sempre atendido pela sua equipe de referência. E a interdisciplinaridade sendo estimulada pelo trabalho em equipe, na interação e integração disciplinar a que se propõe o modelo de agendamento pautado na pessoa e na integralidade do cuidado ${ }^{12,13,15}$.

Pôde-se observar que houve redução nas faltas às consultas após a implantação deste novo sistema de agendamento. O maior poder de resolubilidade e autogestão da agenda podem determinar menos estresse da equipe e mais satisfação dos usuários.,12,13,15 Oportunizar consultas para o mesmo dia pode ser uma forma de humanizar o atendimento. ${ }^{8}$

Analisar o acesso ao atendimento na unidade de saúde a partir da implementação do Acesso Avançado como estratégia de organização da agenda numa UBS do município de Diadema, São Paulo, mostrou aumento no número de usuários atendidos e queda na taxa de faltas dos usuários às consultas. As agendas médicas foram otimizadas e as faltas das consultas programáticas eram aproveitadas para atendimento à demanda espontânea.

Para garantir a ampliação do acesso sem perder a qualidade no atendimento é necessário o equilíbrio entre a oferta e a demanda. O Acesso Avançado pode ser adaptado à realidade local de cada UBS e pode funcionar bem, mesmo se a demanda exceder a capacidade em um determinado dia, mas se a procura exceder a capacidade de atendimento constantemente, nenhum sistema vai funcionar, nem no modelo tradicional, nem no modelo de Acesso Avançado. ${ }^{2,7}$

O excesso de demanda pode levar ao aumento da carga de trabalho, insatisfação e adoecimento dos trabalhadores, impactando no cuidado prestado. Estudos apontam vários aspectos ligados ao aumento da carga de trabalho: excesso de demanda; equipes incompletas; território superestimado; e insuficiência dos recursos humanos. ${ }^{6,20,21}$

Embora a cobertura da ESF venha aumentando, a iniquidade de acesso ainda persiste. Equilibrar a demanda com capacidade ainda parece ser um sério problema a ser enfrentado em relação ao acesso na APS. ${ }^{1,9}$

A abertura da ESF para um acesso facilitado exige um novo pensar e agir dos serviços, exigindo também um novo olhar do Ministério da Saúde, no sentido de revisitar os parâmetros da ESF a fim de torná-la mais acessível ao cidadão, construindo mecanismos asseguradores do acesso. Pouco adiantará a garantia política, legal e institucional que preconize o atendimento qualificado e longitudinal dos usuários se não se rediscutir a proporcionalidade entre equipe e população adscrita. 
Reduzir o número de usuários por equipe e melhorar os processos de trabalho e agendas dos profissionais é necessário para a construção de um equilíbrio entre cuidado à demanda espontânea e das outras ações agendadas e/ou programáticas, com fácil acesso e promovendo longitudinalidade.7,15

Vale ressaltar que a proposta é de um novo modelo de agendamento e de ampliação do acesso e não de um modelo técnico-assistencial. Desta forma, é imperativo que os gestores e equipes se empenhem em discutir medidas capazes de assegurar os princípios, as diretrizes e os fundamentos da ESF, com o objetivo de garantir a integralidade, a gestão do cuidado e a longitudinalidade.

Espera-se colaborar para ampliação das discussões sobre acesso à APS e subsidiar equipes e municípios na decisão de implementar o Acesso Avançado como estratégia de reorganização de suas agendas e de seus processos de trabalho.

Ainda são escassos os estudos nacionais sobre a influência desse modelo no acesso aos serviços da APS no Brasil. Apesar deste relato não permitir generalizações, espera-se contribuir e fomentar discussões acerca da otimização do acesso à APS, suas complexidades e peculiaridades. Com isso, pretende-se também contribuir para promover um movimento das equipes e municípios em estudar e publicar suas experiências de implantação do Acesso Avançado visando ampliar as discussões e promover maior entendimento sobre este fenômeno no contexto brasileiro da APS.

Dados preliminares desse estudo foram apresentados para o Secretário de Saúde do município de Diadema, Coordenação da Atenção Básica, e para os outros gerentes de UBS em reunião de colegiado, que se mostraram sensibilizados para viabilizar a implantação em outras unidades. Com base nesta experiência, o município iniciou um movimento de implantação do Acesso Avançado e disparou a necessidade de criação de um GT (Grupo Técnico) para discutir, apoiar e qualificar esses processos em cada UBS, preparando desde a escolha do modelo, a construção de novos fluxos e agendas, a sensibilização das equipes e as mudanças nos processos de trabalho. Foi criado um documento norteador da implantação para as unidades interessadas e oferecido apoio técnico para qualificar este processo. Atualmente, das 20 UBS do município, o Acesso Avançado está implantada em onze unidades e com projeção futura de ampliação nos próximos meses.

Com a difusão do Acesso Avançado no Brasil e, particularmente, no município de Diadema, propõe-se analisar sua influência sobre os demais atributos da APS, particularmente nos atributos de longitudinalidade e integralidade do cuidado.

\section{Contribuição dos autores}

Contribuições substanciais para a concepção ou delineamento do trabalho, ou a aquisição, análise ou interpretação dos dados: FMSBC, DASF, LYIN, LAF. Elaboração do rascunho do trabalho, ou sua revisão crítica para conteúdo intelectual importante: FMSBC, DASF, LYIN, LAF. Aprovação final da versão a ser publicada: FMSBC, DASF, LYIN, LAF. Concordância em prestar contas de todos os aspectos do trabalho, assegurando que as questões relacionadas à acurácia ou integridade de qualquer parte do trabalho sejam devidamente investigadas e resolvidas: FMSBC, DASF, LYIN, LAF. 


\section{REFERÊNCIAS}

1. Rocha SA, Bocchi SCM, Godoy MF. Acesso aos cuidados primários de saúde: revisão integrativa. Physis. 2016 Mar;26(1):87-111. DOI: http://dx.doi.org/10.1590/S0103-73312016000100007

2. Sistema Único de Saúde (SUS). Secretaria Municipal de Saúde de Curitiba. Saúde da Família. Novas possibilidades de organizar o acesso e a agenda na Atenção Primária à Saúde. Curitiba (PR): SUS; 2014; [acesso 2018 Out 16]. Disponível em: http://www.saude.curitiba. pr.gov.br/images/cartilha\%20acesso\%20avançado\%2005_06_14.pdf

3. Balasubramanian H, Biehl S, Dai L, Muriel A. Dynamic allocation of same-day requests in multi-physician primary care practices in the presence of prescheduled appointments. Health Care Manag Sci. 2014;17(1):31-48. DOI: http://dx.doi.org/10.1007/s10729-013-9242-2

4. Assis MAA, Jesus WLA. Acesso aos serviços de saúde: abordagens, conceitos, políticas e modelo de análise. Ciênc Saúde Coletiva. 2012;17(11):2865-75. DOI: http://dx.doi.org/10.1590/S1413-81232012001100002

5. Brasil. Ministério da Saúde. Portaria de Consolidação № 02, Anexo XXII, de 28 de setembro de 2017. Consolidação das normas sobre as políticas nacionais de saúde do Sistema Único de Saúde. Brasília (DF): Ministério da Saúde; 2017; [acesso 2019 Mai 18]. Disponível em: http://bvsms.saude.gov.br/bvs/saudelegis/gm/2017/prc0002_03_10_2017.htm

6. Lima SAV, Silva MRF, Carvalho EMF, Pessoa EAC, Brito ESV, Braga JPR. Elementos que influenciam o acesso à atenção primária na perspectiva dos profissionais e dos usuários de uma rede de serviços de saúde do Recife. Physis·2015;25(2):635-56. DOI: http://dx.doi. org/10.1590/S0103-73312015000200016

7. Tesser CD, Norman AH. Repensando o acesso ao cuidado na Estratégia Saúde da Família. Saúde Soc. 2014;23(3):869-83. DOI: http:// dx.doi.org/10.1590/S0104-12902014000300011

8. Sant'Ana AM. Pesquisa operativa para adequação das agendas num serviço de Atenção Primária. Rev Bras Med Fam Comunidade. 2017 Jan-Dez;12(39):1-9. DOI: http://dx.doi.org/10.5712/rbmfc12(39)1459

9. Vieira-da-Silva LM, Chaves SCL, Esperidião MA, Lopes-Martinho RM. Accessibility to primary healthcare in the capital city of a northeastern state of Brazil: an evaluation of the results of a programme.J Epidemiol Community Health. 2010;64(12):1100-5.DOI:http://dx.doi.org/10.1136/ jech.2009.097220

10. Vidal TB, Tesser CD, Harzheim E, Fontanive PVN. Avaliação do desempenho da Atenção Primária à Saúde em Florianópolis, Santa Catarina, 2012: estudo transversal de base populacional. Epidemiol Serv Saúde. 2018;27(4):e2017504. DOI: https://doi.org/10.5123/ S1679-49742018000400006

11. Silva AS, Nogueira DA, Paraizo CMS, Fracolli LA. Avaliação da Atenção Primária à Saúde: visão dos profissionais de saúde. Rev Esc Enferm USP. 2014 Aug;48(spe):126-8. DOI: https://doi.org/10.1590/S0080-623420140000600018

12. Murray M, Tantau C. Same-day appointments: exploding the access paradigm. Fam Pract Manage [Internet]. 2000 Set; [citado 2018 Dez 20]; 7(8):45-50. Disponível em: https://www.aafp.org/fpm/2000/0900/p45.html

13. Murray M, Berwick D. Advanced access: reducing waiting and delays in primary care. JAMA. 2003;289(8):1035-40. DOI: https://doi. org/10.1001/jama.289.8.1035

14. National Health Service (NHS). Commissioning a new delivery model for unscheduled care in London. London: NHS; 2011 ; [acesso 2018 Dez 20]. Disponível em: http://www.shiftingthebalance.scot.nhs.uk/downloads/1234865158-Unscheduled\%20care_PCT\%20 commissioning\%20guide\%20for\%20a\%20new\%20delivery\%20model_v1\%200.pdf

15. Norman AH, Tesser CD. Access to healthcare in the Family Health Strategy: balance between same day access and prevention/health promotion. Saúde Soc. 2015;24(1):165-79. DOI: http://dx.doi.org/10.1590/S0104-12902015000100013

16. Secretaria Municipal de Saúde de Diadema. Perfil epidemiológico do município de Diadema. Diadema (SP):Prefeitura de Diadema;2017.

17. Brasil. Ministério da Saúde. Secretaria de Atenção à Saúde. Departamento de Atenção Básica. Acolhimento à demanda espontânea. Brasília (DF): Ministério da Saúde; 2011.

18. Starfield B. Atenção primária: equilíbrio entre necessidade de saúde, serviços e tecnologia. Brasília(DF):UNESCO, Ministério da Saúde;2002.

19. Morgan CL, Beerstecher HJ. Satisfaction, demand and opening hours in primary care: an observational study. Br J Gen Pract. 2011;61(589):498-507. DOI: http://dx.doi.org/10.3399/bjgp11X588475

20. Sousa FOS, Medeiros KR, Gurgel Júnior GD, Albuquerque PC. Do normativo à realidade do sistemaúnico de saúde: revelando barreiras deacesso na rede de cuidados assistenciais. Ciênc Saúde Coletiva.2014;19(4):1283-93. DOI: http://dx.doi.org/10.1590/1413-81232014194.01702013

21. Pires DEP, Machado RR, Soratto J, Scherer MA, Gonçalves ASR, Trindade LL. Cargas de trabalho da enfermagem na saúde da família: implicações no acesso universal. Rev Latino-Am Enfermagem. 2016;24:e2677. DOI: http://dx.doi.org/10.1590/1518-8345.0992.2682 